\title{
Woman WITH Facial AND NECK SWELliNG
}

\author{
Meredith Chiaccio, MSIII and Donna Mscisz Williams, MD
}

\section{Case Presentation}

A 28 year-old Caucasian female, 3.5 months postpartum, presented to the TJUH ED with facial and neck swelling. She was in her usual state of health until approximately 1 month prior to admission when she noted the gradual onset of neck swelling. The neck swelling progressed to her face 4 days prior to admission; swelling in both areas was progressive and increasingly uncomfortable. Two months prior, the patient was treated for sinusitis with a course of antibiotics by an allergist; this treatment was unsuccessful, and she was referred her to an otolaryngologist, who performed a neck ultrasound and lab work. The patient reported that several enlarged lymph nodes were found in her neck. The patient was then sent for a CT of the neck and chest, which showed a mass in her mediastinum. She was planning to follow up with a cardiothoracic surgeon, however she developed symptomatic swelling and intermittent dysphagia. The patient

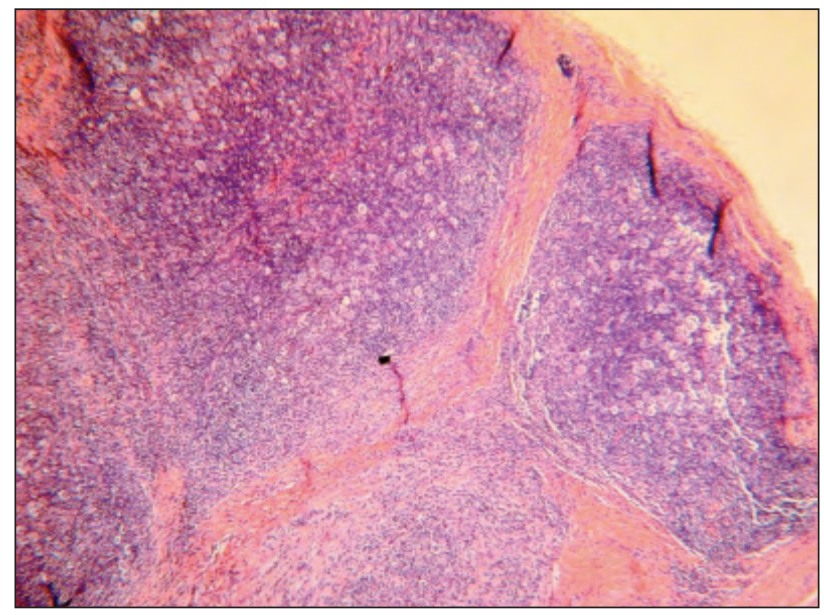

Figure 1. Low power image of mediastinal mass shows nodular demarcation of cells by fibrous bands.

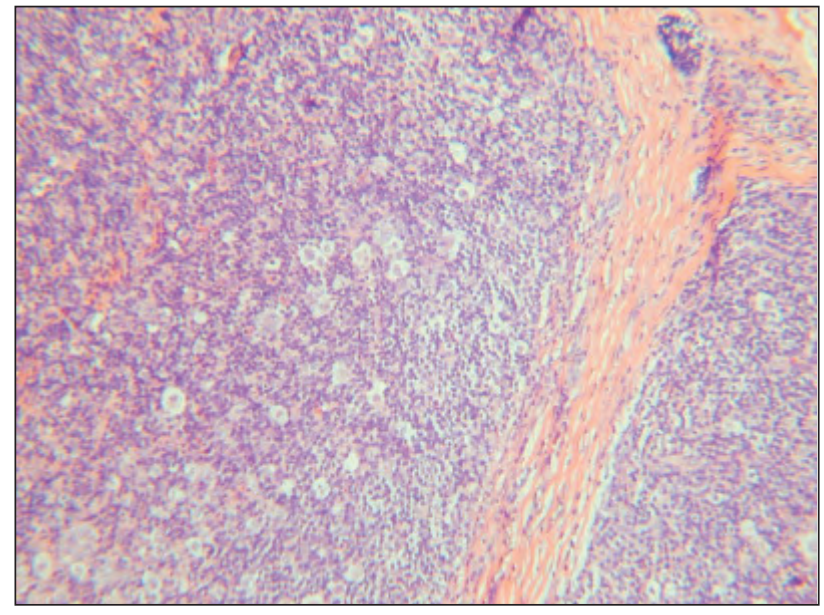

Figure 2. Higher power image showing large multi-nucleated cells with clear cytoplasm described a sensation of choking and strangulation; these sensations came and went at random times. She also reported tolerating only small amounts of soft foods, such as apple sauce, Jell-O, and yogurt. The dysphagia was present only with solid foods, not with liquids.

The patient also complained of dull upper back pain located in her upper right trapezius area for the past month which was exacerbated by holding her child and other physical activities and was alleviated somewhat by ibuprofen; this pain felt like a pulled muscle and she rated it $4 / 10$. She stated that chest pain, located at her right anterior chest, had bothered her for the last 4 days; she described this pain as piercing, sharp, 9/10 in severity, and intermittent, with no exacerbating or ameliorating factors.

The patient's past medical history included intermittent migraines for several years which were well-controlled and a full term

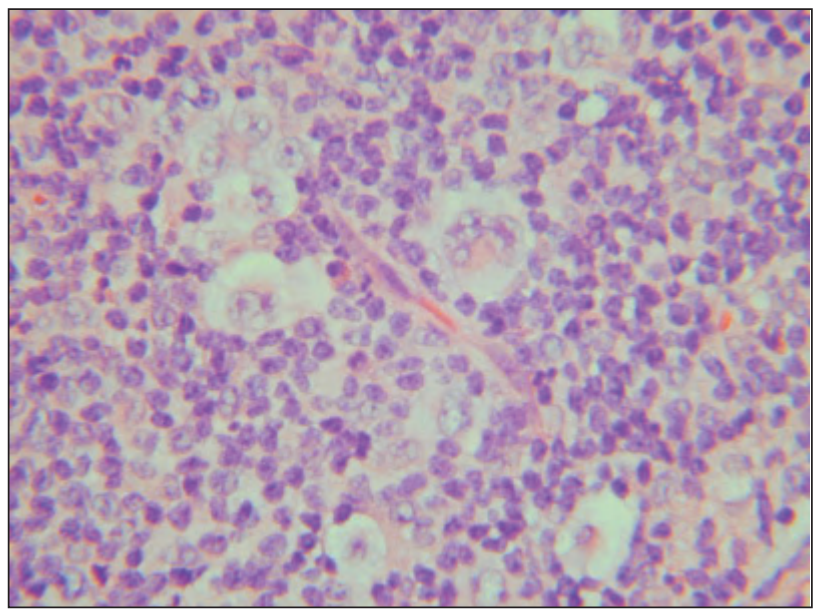

Figure 3. High power image of Reed-Sternberg cells in a mixed inflammatory background.

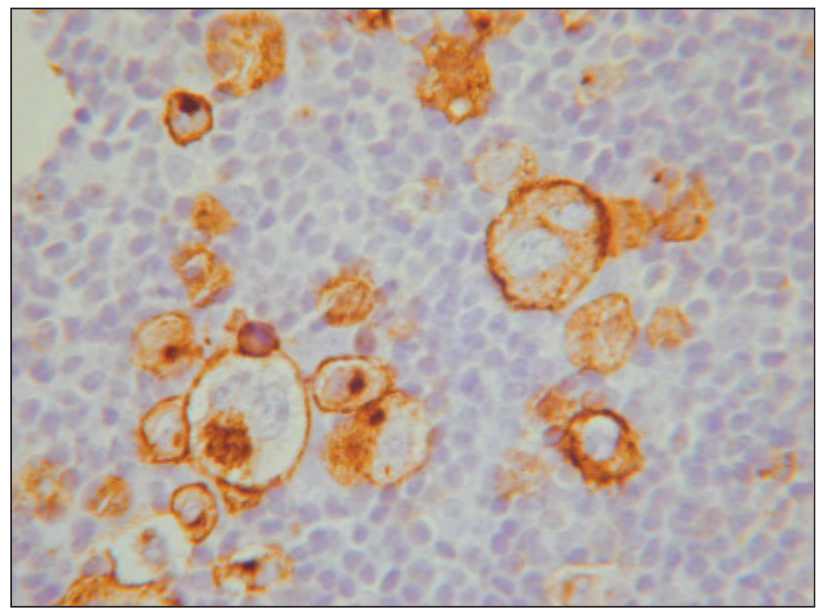

Figure 4. Immunostain showing CD15+ staining of binucleated Reed Sternberg cells. 
uncomplicated vaginal delivery four months prior after which she breast fed for 6.5 weeks. Her surgical history was significant only for appendectomy and breast augmentation. She was taking no medications and reported allergies to penicillin, latex, iodine, and shellfish, which all cause pruritic rash. She denied alcohol, tobacco, or substance abuse. Her family history was noncontributory.

Her review of systems was significant for $7 \mathrm{lb}$ weight gain in the last week, congested and swollen right ear, bilateral sinus pressure, hoarseness, bulging veins which appeared in left neck 1 week ago, and occasional pulsations in her neck bilaterally. She reported edema in the right hand, bilateral swelling of hands and fingers (right greater than left, worsening recently), as well as tingling and numbness in the right fingertips. The patient reported feeling lightheaded when sitting up from supine or when bending over to pick something up off floor, and she felt that her neck swelling worsened when leaning forward. The patient also reported intermittent throbbing headaches, minimally relieved by ibuprofen.

On physical exam, the patient was in no acute distress with stable vital signs. The head and neck exam was significant for an enlarged, well-developed neck with visible tortuous collateral vessels bulging on the left side. Neck circumference was $31.5 \mathrm{~cm}$. Sternocleidomastoids were prominent bilaterally. There was no cervical or supraclavicular lymphadenopathy, nodules, or enlarged thyroid. The patient had maxillary and frontal sinus tenderness to palpation bilaterally, right greater than left. No stridor was appreciated. Generalized distension in neck prevented clear assessment of jugular venous pressure. Pemberton sign was negative. Examination of the lungs revealed decreased breath sounds on right side. Extremity exam revealed dusky/dark appearance of the skin of right extremities and edema of right hand. No axillary or inguinal lymphadenopathy present.

\section{Assessment}

The constellation of this patient's history, signs and symptoms, labs, imaging studies, and knowledge of presence of mediastinal mass were consistent with Superior Vena Cava (SVC) Syndrome. This diagnosis was supported by known presence of anterior superior mediastinal mass with SVC displacement, swelling of the neck and face, development of collateral neck vessels, unilateral cyanosis, swelling and tingling and numbness of the upper extremity, and positional lightheadedness.

\section{Hospital course}

Initial serologic studies were unrevealing. Chest $\mathrm{x}$-ray in the ED revealed a widened superior mediasinum. CT of the chest performed shortly prior to presentation forwarded from an outside institution showed a large homogeneously enhancing anterior mediastinal soft tissue mass insinuating between aortic branch vessels, displacing the SVC laterally with compression. The mass was measured at $9.4 \mathrm{~cm} \times 4.9 \mathrm{~cm} \times 13.8 \mathrm{~cm}$. The patient underwent bronchosopy and transbronchial biopsy of the mediastinal mass, but pathology was indeterminate.
Cardiothoracic surgery was consulted for mediastinoscopy, and biopsies revealed Hodgkin's lymphoma, nodular sclerosing subtype (see Figures 1-4). Both Medical Oncology and Radiation Oncology were consulted, and treatment plan was made. The patient underwent bilateral bone marrow biopsies which were negative. Remainder of the staging work-up was significant only for enlarged lymph nodes in the prevascular and pretracheal spaces. The patient's disease was staged at IIA.

\section{Discussion}

Superior Vena Cava syndrome (SVCS) occurs when the SVC is invaded or externally compressed by a mass or thrombosed. SVCS encompasses a classic constellation of signs and symptoms including facial edema, dyspnea, tachypnea, cyanosis, venous distension, headache, and plethora. Other signs include cough, arm edema, a feeling of head fullness often exacerbated by lying down or bending forward; more seriously, it could present as respiratory distress from tracheal involvement. Pemberton's sign is the development of facial plethora, inspiratory stridor, and non-pulsatile JVP elevation when a patient with SVC syndrome lifts the arms over the head. While dysphagia, hoarseness, and stridor are not included in the definition of SVCS because they result from compression of mediastinal structures other than the SVC (esophagus, laryngeal nerve, and trachea respectively), their presence is negatively correlated with prognosis. Presentation depends on both the location and rate of growth of the mass; growth rate also determines how well the syndrome can be compensated by new collateral circulation. Important collateral veins arise from the azygos, lateral thoracic, paraspinous, and internal mammary veins.

Approximately $85 \%$ of cases of SVCS are caused by malignant tumors; the rest are due to infection and thrombosis. Up to $60 \%$ of patients presenting with SVCS due to underlying malignancy present without a known diagnosis of cancer. The most common malignant causes of SVCS are lung cancer, lymphoma, thymoma, mediastinal germ cell tumors, and solid organ tumors metastatic to the mediastinum. Bronchogenic carcinoma and lymphoma alone account for $94 \%$ of cases of SVC syndrome. Fibrosing mediastinitis accounts for up to $50 \%$ of nonmalignant causes of SVCS. This clinical entity is often caused by excessive host cell response to a prior Histoplasma capsulatum infection; however, other causative infections include aspergillosis, blastomycosis, actinomycosis, tuberculosis, and Bancroftian filariasis. SVC thrombosis can also occur secondary to indwelling central venous catheters and pacemaker leads.

Lung carcinoma is the most common cause of SVCS and does so via compression or direct SVC invasion by tumor or via mediastinal lymphadenopathy. Two to four percent of patients with lung carcinoma develop SVCS during the course of their disease. Up to $20 \%$ of patients with small cell lung cancer develop SVCS because of the central location of these tumors; peripherally arising lung tumors, including adenocarcinoma and large cell carcinoma, are less likely to cause SVC involvement. Small cell 
lung cancer accounts for approximately $40 \%$ of all cases of SVC syndrome due to lung cancer, with squamous cell carcinoma making up another $18 \%$.

Lymphoma, almost always Non-Hodgkin's type, is the second most common cause of SVCS. Approximately 2-4\% of patients with lymphoma develop SVCS, via lymph node enlargement. The most common lymphomas associated with SVCS are diffuse large B-cell and lymphoblastic lymphomas. Hodgkin's lymphoma, despite its common presentation with mediastinal lymphadenopathy, rarely causes SVCS.

In addition to lung carcinoma and lymphoma, thymoma and germ cell tumors are also among the more common malignant causes of SVC syndrome.

\section{Anterior Mediastinal Masses}

Approximately $2 / 3$ of all mediastinal tumors are benign. Anterior mediastinal masses make up $60 \%$ of all mediastinal masses. The most common neoplasias of the anterior mediastinum include the " $4 \mathrm{Ts}$ " - thymoma, thyroid tumor, germ cell tumors (teratomas), and "terrible" lymphomas. Other anterior mediastinal neoplasms include thymic carcinoid, thymolipoma, and parathyroid adenomas. Thymic cyst, lymphangioma, and intrathoracic goiter comprise the non-neoplastic causes of anterior mediastinal masses.

Lymphoma is the second most common primary anterior mediastinal tumor, second only to thymoma, and accounts for $10-20 \%$ of primary mediastinal masses overall. While lymphoma can affect any mediastinal compartment, most are located in the anterosuperior mediastinum and the remainder in the middle mediastinum. These lymphomas typically present with large, bulky tumors, often with involvement of adjacent intrathoracic structures. While Hodgkin's lymphoma represents only $25-30 \%$ of all cases of lymphomas, it is the most common mediastinal lymphoma; the nodular sclerosing subtype is the most common, and it has particular predilection for the anterior mediastinum. Hodgkin's lymphoma will be discussed below. The most common primary mediastinal Non-Hodgkin's lymphomas are large B-cell lymphoma and lymphoblastic lymphoma. Patients with NHLs are typically older than age 55; 85\% present with advanced disease and typically have constitutional ("B") symptoms, generalized lymphadenopathy, and extensive extranodal disease at time of diagnosis. Indolent NHLs, as compared to aggressive NHLs, generally have a more favorable histologic condition, occur nodally, and are more clinically advanced at presentation. Treatment of NHL depends on histologic classification, site of presentation, and disease extent. Patients with indolent NHLs are treated palliatively with radiation and chemotherapy if necessary, as they typically have a more prolonged disease course, are rarely cured, and almost always have recurrence. Patients with aggressive NHLs are treated with combination chemotherapy and radiation, with possible bone marrow transplantation. Negative prognostic factors for NHLs include extensive extranodal disease and large tumor size at presentation, and slow response to treatment.
Hodgkin's Lymphoma: Diagnosis, Staging, and Treatment Hodgkin's lymphoma, first described by Sir Thomas Hodgkin in 1832, is a malignant lymphoma with a bimodal peak incidence in the second and fifth decades, more commonly found in males. Diagnosis of Hodgkin's lymphoma requires presence of the classic Reed-Sternberg cell - with its abundant basophilic cytoplasm and two large nuclei with pale chromatin and distinct eosinophilic nucleoli - in a mixed inflammatory background of reactive lymphocytes, macrophages, plasma cells, eosinophils, and a disrupted nodal architecture with fibrotic stroma. Only recently were Reed-Sternberg cells discovered to be of B-cell origin; though these cells lack B-cell markers such as CD20 and the B-cell receptor, their precursors are definitively germinal center B-cells that escape negative selection and apoptosis by acquisition of a survival advantage through an as yet unknown mechanism. Epstein-Barr Virus is thought to play a role in the malignant transformation of Reed-Sternberg precursor cells by upregulation of antiapoptotic genes and inhibition of the FAS-signalling pathway.

The two main types of HL are classic HL and lymphocytepredominant HL; classic HL is further classified into 4 subtypes, including nodular sclerosing, lymphocyte rich, lymphocyte depleted, and mixed cellularity. Classic HL accounts for $95 \%$ of all cases of HL. This discussion will center around the nodular sclerosing subtype as it is the most common, accounting for nearly $80 \%$ of cases of HL. With a predominance in young, female patients, the nodular sclerosing subtype is characterized by a nodular growth pattern with sclerosing/fibrotic collagenous banding. The pathology report for the patient in the above case stated that "the lymph node architecture appeared effaced by bands of collagen fibrosis, which subdivide the lymph node into smaller nodules." Nodular sclerosing Hodgkin's lymphoma has a unique predilection for the anterior mediastinum, especially the thymus, and may manifest itself as a discrete, lobulated anterior superior mediastinal mass.

The most common clinical presentation of HL is enlarged nontender cervical and/or supraclavicular lymphadenopathy, and bulky mediastinal lymphadenopathy is additionally characteristic of nodular sclerosing HL. Patients with mediastinal involvement tend to be younger than those without mediastinal disease; mediastinal involvement can manifest as chest pain, cough, wheezing, dysphagia, or SVC syndrome, discussed above. At presentation, $25 \%$ of patients with HL have constitutional "B" symptoms of fever (including cyclic Pel-Ebstein curves), weight loss and night sweats. There is also an unusual association between alcohol consumption and lymph node pain or generalized pruritis in patients with Hodgkin's lymphoma. 
The Modified (Cotswold) Ann Arbor Staging Classification is typically used to stage $\mathrm{HL}$

\begin{tabular}{|c|c|c|c|c|c|c|c|}
\hline I & \multicolumn{2}{|l|}{ II } & III & III' & \multicolumn{2}{|l|}{$\| \mathrm{III}^{2}$} & IV \\
\hline $\begin{array}{l}\text { Single node region } \\
\text { or a lymphoid structure } \\
\text { (spleen, thymus, } \\
\text { Waldeyer's ring) or } \\
\text { involvement of a single } \\
\text { extralymphatic site) }\end{array}$ & \multicolumn{2}{|c|}{$\begin{array}{l}\text { Two or more node } \\
\text { regions on the same } \\
\text { side of the diaphragm, } \\
\text { localized contiguous } \\
\text { involvement of only one } \\
\text { extranodal organ or side } \\
\text { and node region on the } \\
\text { same side of the diaphragm }\end{array}$} & $\begin{array}{l}\text { Node regions involved } \\
\text { on both sides of } \\
\text { diaphragm, and/or } \\
\text { involvement of spleen, } \\
\text { or localized contiguous } \\
\text { involvement of only } \\
\text { one extranodal organ } \\
\text { site or both. }\end{array}$ & $\begin{array}{l}\text { With or without } \\
\text { involvement of } \\
\text { splenic, hilar, celiac, } \\
\text { or portal nodes. }\end{array}$ & \multicolumn{2}{|c|}{$\begin{array}{l}\text { With involvement } \\
\text { of para-aortic, iliac, } \\
\text { and mesenteric nodes. }\end{array}$} & $\begin{array}{l}\text { Diffuse or disseminated } \\
\text { involvement of one } \\
\text { or more extranodal } \\
\text { organs or tissues, } \\
\text { with our without } \\
\text { associated node } \\
\text { involvement. }\end{array}$ \\
\hline \multicolumn{8}{|l|}{ Modifiers } \\
\hline \multicolumn{2}{|l|}{$\mathrm{A}$} & \multicolumn{2}{|l|}{ B } & $x$ & & \multicolumn{2}{|l|}{$E$} \\
\hline \multicolumn{2}{|c|}{ constitutional "B" symptoms absent } & \multicolumn{2}{|c|}{$\begin{array}{l}\text { fever }>38^{\circ} \mathrm{C} \text {, weight loss }>10 \% \\
\text { in } 6 \text { months, night sweats }\end{array}$} & $\begin{array}{l}\text { bulky disease (widened } \\
\text { by }>1 / 3 \text { or presence } \\
w / \text { max dimension }>10\end{array}$ & $\begin{array}{l}\text { liastinum } \\
\text { lal mass }\end{array}$ & \multicolumn{2}{|c|}{$\begin{array}{l}\text { involvement of a single extranodal } \\
\text { site that is contiguous or proximal } \\
\text { to the known nodal site }\end{array}$} \\
\hline
\end{tabular}

Anatomic staging helps distinguish patients that would likely benefit from radiation therapy alone from those requiring systemic treatment. Most patients with HL present with disease localized above the diaphragm; approximately $50 \%$ of patients present in stage I or II, and less than $5-10 \%$ have extranodal disease at time of diagnosis. Subtype and stage are the two most important prognostic factors in HL: the nodular sclerosis subtype carries a very good prognosis, while lymphocyte-depleted HL carries the worst prognosis of the HL subtypes. Constitutional symptoms, age greater than 45, extranodal or bulky mediastinal disease, high ESR or $\mathrm{LDH}$, and greater than five splenic disease foci are adverse prognostic factors.

Patients with stage IA, IB, IIA, and IIB disease are treated with combined-modality therapy of combination chemotherapy plus involved-field irradiation. This concept has replaced prior treatment plans of radiation therapy alone, which was associated with a high incidence of complications including gonadal toxicity and secondary malignancies. Combined-modality therapy also provides improved disease control and lower morbidity. Patients with stage III or IV disease receive combination chemotherapy in occasional combination with radiation.

The chemotherapeutic regimen of choice for Hodgkin's lymphoma is ABVD: doxorubicin, bleomycin, vinblastine, and dacarbazine. The MOPP regimen (mechlorethamine, vincristine, procarbazine, prednisone) was the first drug regimen that produced a high proportion of complete remissions in Hodgkin's lymphoma; ABVD was later introduced as a potentially curative salvage regimen and was shown in 1992 to be superior to the MOPP regimen as it is more efficacious and does not cause sterility. A 2003 trial comparing ABVD to a hybrid MOPP/ABV regimen showed that the hybrid regimen caused more life-threatening side effects during the initial treatment phase and had increased incidence of late complications of sterility and secondary malignancies, especially myelodysplastic syndrome and secondary acute leukemia, without any gain of efficacy. Four cycles of ABVD are used in early-stage disease and six to eight are used in advancedstage disease. Relapsed disseminated disease is treated with highdose chemotherapy and autologous bone marrow transplantation.

\section{Follow-up}

The patient described in the above case can expect a 12-year freedom from progression of approximately $94 \%$ and an overall survival rate of $94 \%$.

\section{References}

1. Drews, Reed E. Superior Vena Cava Syndrome. Up-To-Date, version 14.2, accessed September 21, 2006.

2. Macchiarini Paolo, Helmut Ostertag. Uncommon Primary Mediastinal Tumours. The Lancet 5(2004): 107-18.

3. Strollo DC, Rosado de Christenson ML, Jett JR. Primary Mediastinal Tumors, Part 2: Tumors of the Middle and Posterior Mediastinum. Chest 112(5): 1344-1357, 1997.

4. Nayak LM, Deschler DG. Lymphomas. Otolaryngologic Clinics of North America 36(2003): 625-646.

5. Thomas RK, Daniel Re, Jurgen Wolf, Volker Diehl. Part I: Hodgkin's lymphoma molecular biology of Hodgkin and Reed-Sternberg cells. The Lancet 5(2004): 11-18.

6. Dominguez AR, Marquez A, Guma J, Llanos M. Treatment of Stage I and II Hodgkin's lymphoma with ABVD chemotherapy: results after 7 years of a prospective study. Annals of Oncology 15(2004): 1798-1804.

7. Duggan DB, Petroni GR, Johnson JL, Glick JH. Randomized Comparison of ABVD and MOPP/ABV Hybrid for the Treatment of Advanced Hodgkin's Disease: Report of an Intergroup Trial. Journal of Clinical Oncology 21(4): 607-614, 2003.

8. Meyer RM, Ambinder RF, Stroobants S. Hodgkin's Lymphoma: Evolving Concepts with Implications for Practice. American Society of Hematology 2004, 184-202.

Special thanks to Dr. William Kocher of the Department of Pathology for providing images and guidance. 